Proceedings

\title{
Testing New Solutions for Self Inspection and Quality Checks in the Real World Workshop ${ }^{+}$
}

\author{
Jason Page ${ }^{1, *}$ and Manon Brancart ${ }^{2}$ \\ 1 Ingleton Wood LLP, 874 The Crescent, Colchester Business Park, Colchester CO4 9YQ, UK \\ 2 Entreprise Jacques Delens s.a, Col-Vert Avenue 1, 1170 Watermael-Boitsfort, Belgium; \\ mbrancart@jacquesdelens.be \\ * Correspondence: Jason.page@ingletonwood.co.uk \\ + Presented at Sustainable Places 2017 (SP2017) Conference, Middlesbrough, UK, 28-30 June 2017.
}

Published: 8 November 2017

\begin{abstract}
This workshop carries on a series of workshops presented at Sustainable Places in 2015 and 2016 building upon the discussions surrounding the innovation within the project. ACCEPT. ACCEPT is now in its third year meaning that the ideas have been progressed to an advanced stage. This workshop focuses on the emerging technological prototypes and the piloting program including initial results, successes and failures providing an opportunity to have an open discussion with an opportunity for delegates to gain useful insights that may assist other projects.
\end{abstract}

Keywords: performance gap; augmented reality; pilot; prototype

\section{Introduction}

ACCEPT (Assistant for quality check during construction execution processes for energyefficient buildings) is a 3 year research project funded through the EU Horizon 2020 program. ACCEPT, develops an innovative approach to ensure that buildings are built as designed and hence perform as design. The project uses innovative approaches and technologies to facilitate a step change in the way in which construction projects are managed through construction site activities.

ACCEPT consists of 3 software apps to support the construction industry in knowledge transfer and quality assurance to improve energy efficiency of buildings. The Construction Operative (CoOp) App runs on smart glasses guiding construction workers with Augmented Reality; site managers access a tablet app (SiMa) linked to on-site sensors and project data; and a Dashboard links designers to ACCEPT so all users can collaborate in real-time.

This workshop focuses on the emerging technological prototypes and the piloting program including initial results, successes and failures. The workshop consists of 4 parts, the first 3 tell the story of ACCEPT and its piloting process each $20 \mathrm{~min}$ in length. The final part is an opportunity to have an open discussion with an opportunity for delegates to gain useful insights that may assist other projects.

Part 1-Introduction and overview of ACCEPT including the main innovation-20 min presentation-Jason Page (Ingleton Wood).

Part 2-Brief demonstration of the emerging prototypes-20 min presentation-Jason Page (Ingleton Wood) and Manon Brancart (Entreprises Jacques Delens).

Part 3-Explanation of the piloting activities including piloting techniques, any emerging results and successes and failures of the processes-20 min presentation-Manon Brancart (Entreprises Jacques Delens).

Part 4-Open discussion workshop with audience about the use of technology and how far the emerging prototypes meet the brief. $30 \mathrm{~min}$. 


\section{Introduction and Overview of ACCEPT Including the Main Innovation}

ACCEPT is a 3 year European research project from h2020 in its 3rd and final year. As such the concept has been conceived, taken through development up to working prototypes, which are being trialed on pilot sites across Europe. ACCEPT stands for: Assistant for Quality Check during Construction Execution Processes for Energy Efficient Building.

In plain English, ACCEPT is a suite of applications designed for use on mobile devices on the construction site to digitalize processes in a bid to address the performance gap. It is addressing a specific area within the performance gap. Essentially boiled down the performance gap is the difference in energy between what has been calculated and how much energy a building actually uses.

There are many areas in which the performance gap can be influenced, including concept design and planning, detailed design, procurement, construction and commissioning and versification and testing. There are several cross cutting themes including knowledge and skills, responsibility and communication.

ACCEPT is interested in what can be done to improve site processes, homing into the construction and commissioning phases of projects. This is an area that is traditionally overlooked when looking at the performance gap with research focusing on the calculation methodologies and understanding how the end user uses buildings. With the construction phase, there are gaps to fill, however it is not currently known how big they are, but they do amount to the impact that actions throughout a project can have on the final energy use of a building.

These are examples of the sort of problems seen in the construction site that could lead to the determent of the end energy use of a building. From the Zero Carbon Hubs research on reasons behind the performance gap, it can see the sorts of issues that can arise:

- The designer not being able to input on issues. This can often be the case in Design and Build contracts

- The lack of consistency in labour and staffing reducing the benefits of lessons learnt

- Professionals not engaging with each other on site

- Substituting one product for another without knowing what the influence is.

- QA procedures are not generally carried out and responsibility lacking

- Particularly in housing sales teams can agree to changes without knowing the knockon effects to energy

- Proper commissioning standards not being carried out

- Improvisation on site without knowledge of knock on effects

- Installation instructions or product information not available to those installing systems

- No knowledge of proper sequencing of tasks on site

- Lack of knowledge of the impacts of the tasks on the final performance of the building

- Filling out checklists without properly assessing the subject

There are a few issues that need to be worked out but there are some overarching themes, in Knowledge Transfer, Workflow management and QA Processes. Each combines to define the performance gap due to construction site influence.

ACCEPT is looking to address the performance gap so it is directly mapped onto these 3 overarching themes. Firstly the CoOpApp. This is a construction operative app designed to address knowledge transfer. It does this through the use of innovative technology, in the case of ACCEPT, using smart glasses providing agile knowledge transfer including transferring of knowledge between stakeholders specifically targeted at construction workers. Through the innovative use of AR on a construction site, the range of inputs include 3D models, construction details or text/video tutorials delivered to the construction worker as and when they require it.

To address workflow, the SiMaApp (the Site Managers app) for site managers is the main interaction point designed to be used on a tablet computer. The aim of the SiMaApp is to increase the efficiency, reliability and productivity of construction processes by providing interactive work-flow 
between different entities on a construction site. The SiMaApp links to on site sensors to smartly and proactively adjust workflows to optimize processes.

Finally, the Dashboard is a web-based application with the aim of improving the final thermal, acoustic and energy performance of buildings by providing sophisticated quality assurance tools, which is be used actively or passively by stakeholders during the construction process

So ACCEPT is a complete system aimed at plugging the holes in the construction and commissioning phase of construction projects.

\section{Brief Demonstration of the Emerging Prototypes}

The ACCEPT BIM explorer and CoOpApp were demonstrated live with an explanation of the functionality shown and how that will enable address and impact the final performance of the buildings using key success indicators.

Firstly by using sophisticated quality assurance tools, ACCEPT looks to improve the energy performance of the final building, by augmenting the way in which site managers work on construction sites, replacing their analogue processes with digital ones. When a task gets completed the worker will sign off the job as completed. The site manager can then carry out a QA checklist and verify that the task has been undertaken to an acceptable standard. They can use photographs or notes to addend to show good and bad things found.

Using sensors connected smartly to the system to evaluate critical components to spot issues before they become problems. So the ability to connect sensors into the system is important, for example weather sensor can connect to our workflow as a critical element meaning that brickwork tasks are not activated if the weather is too cold. ACCEPT has also developed a method of monitoring concrete curing using thermal/humidity sensors ensuring the quality of concrete whilst potentially speeding up the program.

To reduce the performance gap by using quality assurance tools. That is making sure that the build is completed correctly, first time, in order to ensure that the design intention is carried through to the final building.

Providing accurate and up to date information to the worker when they need it. Information can be supplied directly to construction workers, anything from connected BIM models, to the drawn information, meaning that the worker at point of use can access anything from an installation video to architect's notes, specification or drawn information directly to hand.

By creating an automatic and assessed workflow matrix, an increase in the productivity and reliability of the construction process can be targeted. For a task that may take many days to complete, ACCEPT will identify whether the work is on target or behind target based on the daily completion declaration and verification. The site manger gets the information he needs before the projects runs too far over meaning they can then make the decision to put more workers on or adjust the program to accept a delay.

\section{Explanation of the Piloting Activities Including Piloting Technique, Emerging Results, and Successes and Failures or the Process}

As a European funded project, there are some success indicators in the form of objectives, that the project have signed up to in order to prove the system.

As a project in its final year, the developing prototypes are ready to test in real life scenarios on construction sites across Europe. ACCEPT has already gone through an early pilot phase, taking place in Spain and Belgium testing the early prototypes for the CoOpApp ... h2020 that is the AR application on smart glasses.

The advanced pilot stage takes at the back end of 2017 and runs until near the end of this year, as well as in Spain and Belgium, the UK and Cyprus have pilot sites to test the wider developing system. So far some good qualitative results have been collected, having tested discrete unconnected parts of the ACCEPT system so far. Successes have been found in the feedback from our approach on construction sites. Generally construction site managers and site workers see real benefits from the digitalized workflows, with comments one worker saying that he was autistic and couldn't read 
normal notes on drawings, but a visual representation of those notes with an installation video or images would change the way he use a construction site and hence his job for the better.

Less successful so far, has been the use of smart glasses on site. Our piloting partners have had real concerns about the health and safety aspects of using smart glasses on working construction sites. We have therefore come up with a set of protocols for the use of glasses, including working in an area where no heavy tools are being used, no vehicle movements, not moving whilst wearing the device and being escorted at all times by an observer. Given that some of this contradicts the usefullness of the ACCEPT system, a BIM explorer app has been developed that mimics the usefulness of the application in a portable and less distracting format. The BIM explorer uses google Tango technology using the first consumer device meaning that the ACCEPT system gains the best of both worlds in a portable device and AR technology. The pilots therefore have shaped the delivery methodology of ACCEPT functionality according to real life use by construction workers.

\section{Open Discussion Workshop with Audience about the Use of Technology and How Far the Emerging Prototypes Meet the Brief}

There was a generally consensus that the results so far have been encouraging and that the potential of the innovation to have a lasting impact of the construction industry was good.

Acknowledgments: This project has received funding from the European Union's Horizon 2020 research and innovation programme under grant agreement No. 636895.

Conflicts of Interest: The authors declare no conflict of interest.

(C) 2017 by the authors. Licensee MDPI, Basel, Switzerland. This article is an open access article distributed under the terms and conditions of the Creative Commons Attribution (CC BY) license (http://creativecommons.org/licenses/by/4.0/). 